\title{
Nível de atividade física e quedas acidentais em idosos: uma revisão sistemática
}

\author{
Level of physical activity and accidental falls in elderly: \\ a systematic review
}

1 Universidade Federal de Mato Grosso. Doutoranda do Programa de PósGraduação Interinstitucional em Enfermagem. Cuiabá, MT. Brasil.

2 Universidade Federal de Lavras. Departamento de Educação Física. Lavras, MG. Brasil.

3 Universidade Federal de Mato Grosso. Departamento de Enfermagem. Cuiabá, MT. Brasil.

4 Universidade Federal de São Paulo. Departamento de Enfermagem. São Paulo, SP. Brasil.

Recebido em 27/03/08 Revisado em 22/04/08 Aprovado em 23/06/08
Resumo - O objetivo do presente estudo foi analisar pesquisas que avaliaram a relação entre o nível de atividade física e a ocorrência de quedas em idosos. O método adotado foi uma revisão sistemática da literatura realizada nas bases de dados LILACS e MEDLINE. Como critérios de inclusão, foram selecionadas publicações com população de estudo abrangendo indivíduos com 60 anos ou mais, publicações do tipo artigo em periódicos em texto completo nas bases de dados, nos idiomas Português, Inglês e Espanhol. Foram selecionados 29 documentos, dos quais cinco foram encontrados na base LILACS (1982 a 2007), dois na MEDLINE (1966 a 1996) e 22 documentos na MEDLINE (1997 a 2007). Foram utilizados dez artigos (35\%), sendo que 19 documentos (65\%) foram excluídos por não atenderem aos critérios de inclusão previstos. Dos estudos utilizados $(\mathrm{n}=10)$, a maioria era composta por estudos transversais (60\%). Os estudos encontrados sugerem uma associação entre nível de atividade física e fatores associados à ocorrência de quedas, como por exemplo, capacidade funcional, qualidade de vida e independência nas atividades da vida diária. Pôde-se também observar, em alguns estudos, que o evento da queda levou à restrição de atividade física. Devido à heterogeneidade dos estudos, principalmente em relação a diferentes instrumentos com conteúdos diversificados que dificultaram o pareamento dos resultados, não foi possível identificar se melhor nível de atividade física é capaz de auxiliar na redução da incidência de quedas em idosos.

Palavras-chave: Exercício; Acidentes por quedas; Idoso.

Abstract - The aim of this study was to analyze studies evaluating the association between the level of physical activity and the occurrence of accidental falls in the elderly. A systematic literature review of the LILACS and MEDLINE databases was conducted. As inclusion criteria, complete scientific articles investigating subjects older than 60 years, published in Portuguese, English or Spanish, were selected. Twenty-nine articles were retrieved, five from LILACS (1982 to 2007), two from MEDLINE (1966 to 1996), and 22 from MEDLINE (from 1997 to 2007). Ten (35\%) articles were selected based on the inclusion criteria and 19 (65\%) were excluded. A cross-sectional design was the most frequent type of study (60\%). The studies identified suggest an association between the level of physical activity and factors related to the occurrence of falls such as functional disability, quality of life and independence to perform daily activities. Falls were found to restrict physical activity in some studies. In view of the heterogeneity of the studies in terms of methods and assessments, it was not possible to determine whether a better level of physical activity is able to decrease the incidence of falls in the elderly.

Key words: Exercise, Accidental falls, Aged. 


\section{INTRODUÇÃO}

A proporção de idosos que sofrem queda acidental, em um período de um ano, varia de 28 a $35 \%$ para aqueles com idade entre 65 e 74 anos e, de 32 a $42 \%$ para aqueles com mais de 75 anos $^{1}$. Esses dados concordam com os achados de Perracini e Ramos $^{2}$ em um estudo de seguimento de dois anos, no qual 30,9\% dos idosos sofreram queda no ano anterior ao inquérito. Desses, 10,8\% relataram duas ou mais quedas no período. Estes episódios, mesmo não resultando em fraturas e internações, podem levar a um posterior medo de cair ${ }^{3}$.

A queda é um complexo fenômeno de origem multifatorial que tem relação principalmente com a falta de equilíbrio e pobre desempenho físico ${ }^{4}$. Por esse motivo, um bom nível de atividade física pode ser considerado fator protetor para a ocorrência de quedas.

A atividade física pode ser definida como qualquer movimento corporal voluntário, realizado pela musculatura esquelética que resulta em um gasto energético acima dos níveis de repouso ${ }^{5}$. Neste sentido, praticar atividades físicas não é somente se engajar em exercícios ou esportes, mas executar atividades cotidianas como caminhar, subir escadas ou limpar a casa.

Pesquisas mostram que idosos que participam de programas de exercício para prevenção de quedas melhoram ou mantêm seus níveis de força, equilíbrio e mobilidade, embora tenha sido verificado que intervenções de prática de exercícios em grupo apresentam efetividade desconhecida ${ }^{6}$.

No idoso, as quedas acontecem tanto dentro de casa quanto em ambientes externos, mas a sua ocorrência depende das condições de risco do ambiente em que o idoso se expõe. Em um estudo de Northridge et al. ${ }^{7}$, que comparou o número de quedas sofridas, dentro de casa, por idosos frágeis e considerados vigorosos (divididos conforme seus escores em uma escala de fragilidade baseada em níveis de equilíbrio e desempenho de marcha), observou-se que os frágeis caem duas vezes mais, e que os vigorosos sofrem quedas associadas aos riscos do ambiente. Idosos ativos podem estar mais expostos a situações de perigo no que se refere aos espaços externos. Por exemplo, pessoas que relatam fazer mais atividades físicas nas horas de lazer apresentam maior risco de queda em ambientes externos ${ }^{8}$.

Finalmente, a relação entre nível de atividade física e quedas aparece como uma questão relativamente controversa, pois estudos prévios mostram que a atividade física freqüente pode ser considerada um fator de risco para quedas enquanto que a diversidade de atividade física é considerada como fator protetor?.

O objetivo da presente pesquisa foi fazer um levantamento bibliográfico sistematizado para analisar pesquisas que avaliaram a relação entre o nível de atividade física e a ocorrência de quedas em idosos.

\section{PROCEDIMENTOS METODOLÓGICOS}

Trata-se de uma revisão de trabalhos científicos que estudaram a relação entre quedas acidentais em idosos e sua associação com o nível de atividade física, seja por via da influência desta na ocorrência da queda, ou pelo nível de atividade física como uma variável estudada. Para isso, utilizaram-se as bases de busca LILACS (1982 a 2007), MEDLINE no período de 1966 a 1996 e MEDLINE no período de 1997 a 2007.

Os termos de pesquisa foram inseridos no campo de pesquisa das bases de dados da seguinte maneira: queda $\$$ idoso $\$$ nível atividade $\$$ física $\$$ ou fall aged physical activity level. Nas bases, o símbolo $\$$ equivale ao (OR) e quer dizer, queda (ou quedas), idosos (ou idosas, ou idoso, ou idosa) e nível de atividade física (ou atividades físicas). A busca foi conduzida em 2007, por dois dos pesquisadores responsáveis pelo estudo, de forma independente e cegada, obedecendo aos critérios de inclusão definidos no protocolo de pesquisa. As discordâncias neste aspecto foram resolvidas por consenso.

\section{Critérios de inclusão}

Somente foram selecionadas publicações do tipo artigo em periódicos (Journal Articles), estudos do tipo coorte, caso-controle, transversais, longitudinais, ensaios clínicos/estudos controlados randomizados nos idiomas Inglês, Espanhol e Português, disponíveis em texto completo. Foram selecionados artigos que incluíssem pessoas consideradas idosas de acordo com as leis nacionais ${ }^{10}$ (60 anos ou mais).

\section{RESULTADOS}

A busca bibliográfica resultou em 29 documentos, dos quais cinco foram encontrados na base LILACS, dois na MEDLINE - 1966 a 1996 e 22 na MEDLINE - 1997 a 2007.

Em consonância com os critérios de inclusão, permaneceram dez artigos para a análise (35\%), sendo que 19 documentos (65\%) foram excluídos pelos motivos descritos a seguir. 
Tabela 1. Desenho dos estudos e suas características quanto ao ano, país de publicação, delineamento, tipo de amostra, idade e sexo dos participantes.

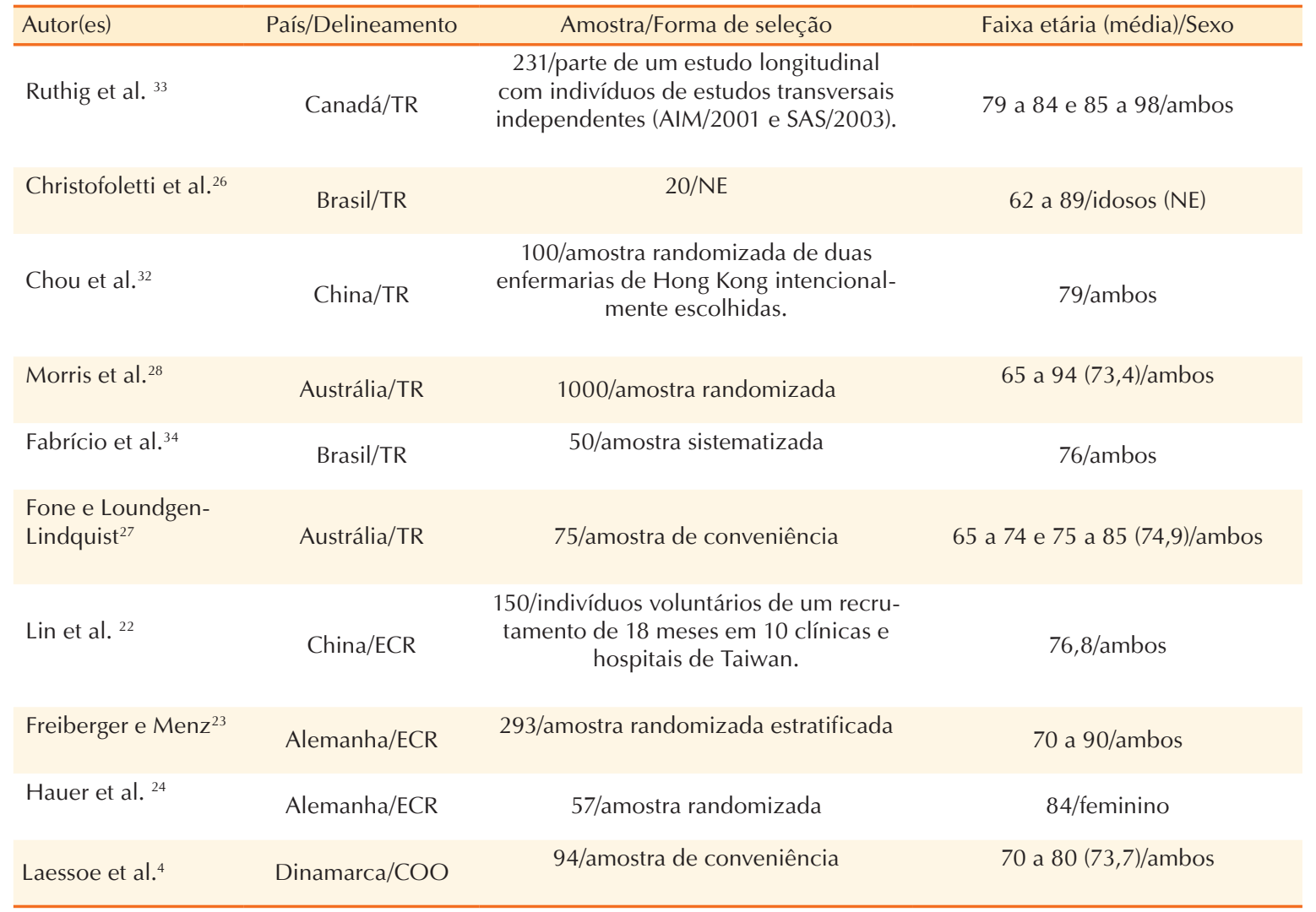

$\mathbf{T R}=$ Estudo transversal, $\mathbf{E C R}=$ Estudo controlado randomizado, $\mathbf{C O O}=$ Coorte/segmento, NE= Não especificado, $\mathbf{A I M}=$ Aging in Manitoba project, $\mathbf{S A S}=$ Successful Aging Study.

Cinco documentos foram excluídos pelo tipo de publicação, sendo um capítulo de livro ${ }^{11}$, uma tese ${ }^{12}$ e três estudos de revisão, ${ }^{9,13}$. Os artigos de revisão serviram como apoio para a base introdutória do presente trabalho. Três artigos foram excluídos por estarem em outros idiomas (um em japonês ${ }^{15}$, um em alemão ${ }^{16}$, e um em francês ${ }^{17}$ ). Quatro artigos foram excluídos por não se relacionarem com o tema ${ }^{3,18-20}$. Um estudo de caso $^{21}$ foi excluído devido à idade da participante pesquisada, ser inferior a 60 anos. Finalmente, seis artigos foram excluídos por não estarem disponíveis em texto completo.

Em relação ao delineamento dos estudos selecionados $(n=10)$, seis caracterizam-se como estudos transversais (TR), três como estudos controlados randomizados (ECR) e um como estudo de Coorte (COO). Portanto, a maioria foi composta por TR (60\%). Destes, dois foram realizados no Brasil, dois na Austrália, um no Canadá e um na China (Tabela 1).

Nos TR, verificamos uma grande variedade de parâmetros avaliados, desde aspectos físicos e psicológicos até aspectos relacionados à saúde e qualidade de vida, bem como de uma diversificada lista de instrumentos utilizados para a avaliação destes parâmetros (Tabela 2).

Os ECR propuseram intervenções com exercícios físicos padronizados e os compararam com outros tipos de intervenção. Lin et al..$^{22}$ compararam três programas de prevenção de queda: 1) Educação, 2) Avaliação e modificação para segurança em casa e 3) Treinamento de exercício, em um período de quatro meses. Freiberger e Menz $z^{23}$ compararam três intervenções (Intervenção Multifacetada, Intervenção psicomotora e Exercício físico) com uma situação controle, durante 12 meses. Um estudo de Hauer et al. ${ }^{24}$ se diferenciou por fazer um seguimento por dois anos, após os três meses de intervenção. Neste, a comparação foi um treinamento de resistência de alta intensidade progressiva com uma atividade placebo (alongamento ou jogos de salão predominantemente na posição sentada). Ambos os grupos, em função dos problemas ortopédicos, resultantes de quedas ou de cirurgias pelos participantes, também receberam sessões fisioterápicas durante o estudo (Tabela 3).

O objetivo do estudo COO de Laessoe et al. ${ }^{4}$, com seguimento de um ano, foi desenvolver uma 
Tabela 2. Características dos Estudos Transversais quanto a objetivos, ao instrumento de avaliação do nível de atividade física e das demais variáveis.

\begin{tabular}{|c|c|c|c|}
\hline Autor(es) & Objetivo (s) & $\begin{array}{l}\text { Instrumento de medida } \\
\text { de NAF }\end{array}$ & $\begin{array}{l}\text { Instrumentos de medida } \\
\text { e/ou variáveis avaliadas }\end{array}$ \\
\hline Ruthig et $a{ }^{\beta 3}$ & $\begin{array}{l}\text { Verificar o impacto da queda na saúde } \\
\text { física, emoções negativas e atividade física } \\
\text { em idosos. }\end{array}$ & PA, PAR & $\begin{array}{l}\text { CMS, Quedas, PCE, } \\
\text { LOT, GPH, EN }\end{array}$ \\
\hline Christofoletti et al. ${ }^{26}$ & $\begin{array}{l}\text { Avaliar o risco de queda em idosos (com } \\
\text { Parkinson, Alzheimer e saudáveis). Relação } \\
\text { entre risco de queda e nível de atividade } \\
\text { física. }\end{array}$ & QBMI & EEFB, TUG, MEEM \\
\hline Chou et al. ${ }^{32}$ & $\begin{array}{l}\text { Examinar a relação entre medo de cair e } \\
\text { sintomas depressivos em idosos; e a partici- } \\
\text { pação em atividades físicas e auto-eficácia } \\
\text { de queda e sua ligação com as variáveis } \\
\text { anteriores. }\end{array}$ & Alguns itens do SAFE & GDS, SAFE, FES \\
\hline Morris et al. ${ }^{28}$ & $\begin{array}{l}\text { Relatar a visão das pessoas idosas sobre } \\
\text { porque elas caem, bem como o perfil dessas } \\
\text { pessoas, considerando condições médi- } \\
\text { cas, caidores freqüentes com ocasionais, e } \\
\text { comparando-os com não caidores. }\end{array}$ & $\begin{array}{l}\text { FAF (baseada no } \\
\text { RFPMC) } \\
\text { AIVD }\end{array}$ & $\begin{array}{l}\text { CMS (baseada na } \\
\text { EPESE), FD, UM, MOB, } \\
\text { PV, MC }\end{array}$ \\
\hline Fabrício et al. ${ }^{34}$ & $\begin{array}{l}\text { Identificar o local, causas e conseqüências } \\
\text { da queda em idosos atendidos em hospital } \\
\text { público. }\end{array}$ & AIVD & $\begin{array}{l}\text { Restrições na capacida- } \\
\text { de funcional nas ABVD }\end{array}$ \\
\hline $\begin{array}{l}\text { Fone, Loundgen- } \\
\text { Lindquist }^{27}\end{array}$ & $\begin{array}{l}\text { Comparar o desempenho em testes físicos e } \\
\text { funcionais em dois grupos de idade ( } 65-74 \text { e } \\
75 \text { a } 85 \text { anos) avaliando também saúde auto- } \\
\text { relatada, capacidade de dirigir veículos, que- } \\
\text { das e envolvimento com ocupações físicas. }\end{array}$ & PASE & $\begin{array}{l}\text { HSOP (inclui quedas), } \\
\text { MFCQ, TCF, Dirigir } \\
\text { veículo. }\end{array}$ \\
\hline
\end{tabular}

QBMI = Questionário Baecke Modificado para Idosos, EEFB = Escala de Equilíbrio Funcional de Berg, TUG = Timed Up and Go test, MEEM = Mini-Exame do Estado Mental, AIVD = Atividades Instrumentais da Vida Diária (ex. subir escadas), ABVD = Atividades Básicas da Vida Diária (ex. alimentar-se), PCE $=$ Perceived Control Scale, $\mathbf{L O T}=$ Life Orientation Test $(8$ itens sobre otimismo), GPH = General Physical Health (saúde física geral auto-relatada) PA = Physical Activity (2 itens sobre quanto à pessoa se considera ativa comparando-se com outra e como foi sua prática de atividade física - AF - nos últimos meses), PAR = Physical Activity Restriction (21 condições de saúde de restrição à AF), EN = Emoções Negativas (questões sobre os últimos 2 dias), GDS = Geriatric Depression Scale-Short Form, SAFE = Survey of Activities and Fear of Falling in the Elderly (22 itens de medida de medo de cair, incluindo ABVD, AIVD, mobilidade e atividade social), $\mathbf{F E S}=$ The Fall Efficacy Scale, FAF = Freqüência de Atividade física baseada na Risk Factor Prevalence Management Committee RFPMC (3 tipos de enérgicas ou moderadas AF realizadas nas últimas 2 semanas), $\mathbf{C M S}=$ Condições Médicas e de Saúde baseada na Established Populations for Epidemiologic Studies of the Elderly - EPESE (condições como colesterol alto, diabetes, câncer, etc.), FD = Freqüência de Dor (dor persistente limitante de atividades nos últimos 12 meses), $\mathbf{U M}=$ Uso de Medicamento (prescrição nas últimas 2 semanas), $\mathbf{M O B}=\mathbf{M o b i l i d a d e}$ (dificuldade em caminhar $1 \mathrm{Km}$ em superfície plana), PV = Prejuízo Visual (auto-relatado), $\mathbf{M C}=\mathbf{M e d o}$ de cair, HSOP = Health Status of Older Persons' questionnaire (modificado), MFCQ = Modified Functional Capacity Questionnaire , PASE = Physical Activity-based Scale for the Elderly, TCF = Testes de Capacidade Física (Resistência e eficiência no caminhar, função de membros superiores, simulação de subir e descer degraus de transportes públicos, preensão manual).

ferramenta para avaliar parâmetros fisiológicos relacionados a equilíbrio e queda de idosos fisicamente ativos. Para isso, nove testes específicos (equilíbrio estático, habilidade de passadas, função física geral, tempo de reação, força nas pernas, controle postural, variabilidade de marcha, ritmo de marcha e visão) foram selecionados para uma bateria de testes que abrangessem aspectos do desempenho físico que poderiam estar relacionados com o risco de queda. Durante o seguimento, 15\% dos idosos experimentaram pelo menos uma queda. Os grupos de "caidores" e de "não caidores" não foram significativamente diferentes nas variáveis idade, saúde auto-estimadas, confiança no equilíbrio e nível de atividade física. Apenas no teste de equilíbrio estático o grupo dos "caidores" mostrou-se inferior. Neste estudo, observou-se que a queda acontecia nos momentos que a pessoa, mesmo sendo considerada saudável e fisicamente ativa, desafiava seus limites. Por exemplo, muitas quedas aconteceram ao "jogar futebol com os netos", ou quando o indivíduo estava sob efeito de ingestão alcoólica. Os que não sofreram queda evitavam desafiar seu equilíbrio, reduzindo a velocidade das passadas ou evitando caminhar em dias muito frios. Pôde-se então concluir que não é possível predizer o risco de queda apenas avaliando desempenho físico. 
Tabela 3. Características dos Estudos Controlados Randomizados segundo seus objetivos, tipo de intervenção, freqüência e duração do experimento.

\begin{tabular}{|c|c|c|c|c|}
\hline Autor(es) & Objetivo (s) & Intervenção & Freqüência & $\begin{array}{l}\text { Duração } \\
\text { (meses) }\end{array}$ \\
\hline Lin et al. ${ }^{22}$ & $\begin{array}{l}\text { Verificar o efeito de três programas de } \\
\text { prevenção de queda sobre a qualidade } \\
\text { de vida, equilíbrio funcional e marcha, } \\
\text { atividades da vida diária, medo de cair e } \\
\text { depressão em idosos. }\end{array}$ & $\begin{array}{l}\text { 1) ED } \\
\text { 2) HSAM } \\
\text { 3) ET }\end{array}$ & $\begin{array}{l}\text { 1) } 30-40 \mathrm{~min} \\
\text { 2) } 30-40 \mathrm{~min}(1 \stackrel{a}{\mathrm{a}} \cdot \mathrm{sem}) \\
\text { 3) } 40-60 \mathrm{~min} / 3 x / \mathrm{sem}\end{array}$ & 4 \\
\hline Hauer et al. ${ }^{24}$ & $\begin{array}{l}\text { Avaliar força, desempenho funcional, } \\
\text { parâmetros psicológicos relacionados à } \\
\text { queda e atividade física em pacientes geri- } \\
\text { átricos de um ambulatório após três meses } \\
\text { de intervenção. }\end{array}$ & $\begin{array}{l}\text { 1) } \mathrm{PRT}+\mathrm{PHT} \\
\text { 2) } \mathrm{PA}+\mathrm{PHT} \\
\text { 3) } \mathrm{PHT}\end{array}$ & $\begin{array}{l}\text { 1) e 2) } 60 \mathrm{~min} / 3 x / \mathrm{sem} \\
\text { 3) } 25 \mathrm{~min} / 2 \mathrm{x} / \mathrm{sem}\end{array}$ & 3 \\
\hline
\end{tabular}

ED = Educação - Visita social para informação (panfletos de prevenção de queda, alongamento, exercícios de força, cuidados ao caminhar). HSAM = Avaliação e modificação da segurança no lar - 14 modificações realizadas na primeira semana (ex., reparação de baixa iluminação, cadeiras instáveis). ET = Treinamento de exercício - Alongamento, força muscular e equilíbrio com dificuldade gradativa. $\mathbf{M I}=$ Intervenção Multifacetada - treinamento de força, cognitivo, de competência (ênfase na consciência de fatores de risco de perigos ambientais e de medicação) e redução de medo da queda. $\mathbf{P I}=$ Intervenção psicomotora - melhora da interação entre percepção e ação, incorporando coordenação motora, treinamento de competência e treinamento perceptual. $\mathbf{F I}=$ Fitness Intervention - focalizou recursos para saúde física (como força, resistência e flexibilidade) incorporando exercícios a serem feitos em casa, e recomendações de atividades físicas. $\mathbf{C G}=$ Grupo controle - sem intervenção. PRT = Treinamento de resistência progressiva. $\mathbf{P A}=$ Atividade Placebo $\mathbf{P H T}=$ Fisioterapia.

\section{DISCUSSÃO}

Na presente revisão, deu-se ênfase aos ECR devido à hierarquia que classifica as pesquisas quando se busca níveis de evidência (a eficácia de uma intervenção ou de algum tratamento em pesquisas publicadas). No ápice desta hierarquia estão as revisões sistemáticas com ou sem metanálise de Ensaios Clínicos Aleatórios (ECA) e de estudos experimentais, seguidas de ECA, Estudo de Coorte, Caso-controle, Estudos quase-experimentais, Estudos descritivos, Experimental de caso único/série de casos, até a Opinião de experts/Relato de caso ${ }^{25}$.

Para que os estudos selecionados fossem comparados, foi necessário analisar aspectos do método adotado por cada um deles, no que se refere, principalmente, à característica dos participantes das amostras, bem como a maneira como estas foram selecionadas.

A utilização de amostras reduzidas ou não selecionadas aleatoriamente implica estudar uma quantidade de pessoas não representativa de sua população. Amostras de conveniência ou a não especificação de como foram selecionadas apareceram em dois dos seis TR ${ }^{26,27}$. Nos TR, a menor amostragem contava 20 indivíduos ${ }^{26}$ enquanto a maior contava 1000 indivíduos (selecionados de maneira randomizada ${ }^{28}$. As amostras dos ECR foram aleatórias, no $\mathrm{COO}$ foi por conveniência.

Quanto à proporção por gênero, em sete estudos, a proporção de mulheres foi maior, variando de diferenças sutis $(51 \%)^{22}$ a grande maioria $(74 \%)^{4}$. No estudo de Hauer et al. ${ }^{24}$, apenas mulheres foram pesquisadas e, no de Christofoletti et al. ${ }^{26}$, os autores não deixaram claro, apenas citam "idosos”. Já no trabalho de Freiberger e Menz $z^{23}$, 66\% eram homens. A predominância feminina nas amostragens era esperada, considerando a questão da longevidade do sexo feminino em relação ao sexo masculino. Observa-se que as mulheres vivem quatro a dez anos a mais que os homens em todo o mundo ${ }^{29}$. No Brasil, esta proporção é mais expressiva, quanto mais idoso for o segmento, e isto se deve à mortalidade diferencial por sexo ${ }^{30}$.

Devido à facilidade de sua administração e de seu baixo custo, questionários padronizados e validados, instrumentos indiretos que estimam o nível de atividade física (NAF) são comumente aplica$\operatorname{dos}^{30}$. Na literatura, entre 1966 a 2003, pelo menos 12 questionários de atividade física específicos para idosos podem ser encontrados em publicações indexadas em importantes bases de dados?.

A especificidade do instrumento destinado a um determinado grupo etário é um ponto im- 
portante a ser levado em consideração, já que os resultados do mesmo devem ser adequados para identificar o NAF próximo do real. Dos TR analisados, dois estudos utilizaram instrumentos específicos para a população idosa, o $\mathrm{PASE}^{27} \mathrm{e}$ o QBMI - Questionário de Baecke Modificado para $\operatorname{Idosos}^{26}$. Em dois estudos foram considerados subitens de escalas padronizadas como a SAFE Survey of Activities and Fear of Falling in the Elderly ${ }^{31}$ e a RFPMC - Risk Factor Prevalence Management Committee $^{28}$. Uma pesquisa de Ruthig et al. ${ }^{32}$ usou questões soltas para avaliar atividade física e 21 questões que abordam condições de saúde que restringem a sua prática. Baseando-se na definição de atividade física, considerou-se o estudo de Fabrício, Rodrigues e Costa Júnior ${ }^{33}$ quando aborda as atividades instrumentais da vida diária, como subir escadas e caminhar fora de casa.

Em quatro estudos TR ${ }^{27,31.33}$,o NAF foi considerado como uma variável dependente. Nestes estudos, a ocorrência de queda foi relatada pelos participantes nos últimos 12 meses $^{27,32}$ ou seis me$\mathrm{ses}^{31} \mathrm{e}$ um estudo não considerou o tempo, mas a quantidade de pessoas atendidas em um hospital pela causa externa "quedas" no ano de 2000 (CID10 W00-W19) 33 .

No estudo de Ruthig et al..$^{32}$,verificou-se que a queda afeta desfavoravelmente a saúde, o emocional e as respostas de atividade física de idosos com mais de 85 anos em maior extensão do que em idosos mais jovens, devido ao impacto diferencial nas suas respectivas percepções de controle e otimismo. Enquanto o evento da queda predisse maiores restrições na atividade física, a percepção de controle e o otimismo predisseram menor restrição. Essa restrição também apareceu no estudo de Chou et al..$^{31}$, mas em relação ao medo de cair. Por outro lado, embora Fone et al..$^{27}$ tenham observado que 27\% dos idosos que sofreram queda declararam que seu medo de cair surtiu efeito sobre suas atividades físicas, também constataram altos níveis de atividade física em pessoas que sofreram queda. Entretanto, uma correlação estatística não foi realizada devido ao insuficiente número de indivíduos $(\mathrm{n}=20)$.

Fabrício et al. ${ }^{33}$ verificaram que a maioria dos idosos que sofreram queda era do sexo feminino, que $54 \%$ deles sofreram quedas anteriores e que a maioria delas aconteceu dentro de casa (66\%). O evento da queda prejudicou atividades motoras como "caminhar em superfície plana", "caminhar fora de casa" e "subir escadas", bem como atividades consideradas úteis para a independência nas ações do dia-a-dia (como "tomar banho" e "utilizar transportes públicos"). Segundo os autores, depois da queda houve um aumento da dificuldade e dependência do idoso. Então, pode-se observar que o evento da queda afetou o NAF nos estudos analisados, principalmente, em relação à restrição de atividade física.

Dois estudos ${ }^{26,28}$ analisaram o NAF como variável independente. No estudo de Christofoletti et al. ${ }^{26}$, o risco de queda em idosos foi estipulado pela análise de dois testes físicos e pelo Mini-Exame do Estado Mental (MEEM). Idosos saudáveis foram analisados como grupo controle (GC), sendo comparados com idosos com Parkinson (DP) e com demência de Alzheimer (DA). Foram encontrados maiores riscos de queda para os idosos DP, seguido de DA e GC, mas quanto ao NAF, o grupo DP apresentou maiores índices. Contudo, esses achados devem ser observados, considerando o fato da pesquisa ser do tipo observacional, com amostra reduzida e selecionada de maneira não especificada, vieses que podem ter afetado tais resultados. Já no trabalho de Morris et al. ${ }^{28}$ constatou-se que 29\% dos idosos caíram uma ou mais vezes em 12 meses. Destes, 19\% eram "caidores ocasionais" (CO) e 10\% "caidores múltiplos" (CM). Os CO eram mulheres com múltiplas condições médicas, história anterior de dor nas costas e as causas da queda relatadas por elas foram: perda de equilíbrio, falta de atenção e a tontura. Os CM eram mulheres mais velhas e que além das causas citadas pelos $\mathrm{CO}$, relataram dificuldades visuais, distúrbios de mobilidade e quedas fora de casa. A participação em atividades físicas moderadas e vigorosas não influenciou o número de quedas sofridas, contudo, os CM expressaram mais medo de cair e foram mais propensos a restringir suas atividades por esse motivo, embora essa tendência não tenha sido estatisticamente significativa.

Portanto, os dados encontrados até o momento se mostram insuficientes para conclusões sobre o efeito do NAF no risco de queda. Entretanto, algumas evidências puderam ser obtidas pelos ECR. No estudo de Lin et al. ${ }^{22}$, três intervenções foram aplicadas durante quatro meses para avaliação da qualidade de vida - QV (nos domínios físico, psicológico, social e ambiental), equilíbrio funcional, medo de cair e depressão. $\mathrm{O}$ grupo Treinamento de Exercício (ET) apresentou melhora nos quatro domínios da QV e nas demais variáveis estudadas, exceto na depressão. Foi verificada a incidência de queda, seis meses após o término das intervenções e não foram constatadas diferenças significativas entre os grupos. Tais resultados podem dever-se 
à característica inicial da amostra, ou seja, índice baixo de queda (13\% em 12 meses) e alto em atividade física (58\% exercitavam-se regularmente) em comparação com as amostras de outros estudos. Nesse caso, o período de intervenção pode ter sido insuficiente para se chegar a uma prevenção. Além disso, a ausência de um grupo controle prejudicou a avaliação das intervenções quanto a esse aspecto. Contudo, concluiu-se que houve superioridade do ET sobre as outras intervenções no que diz respeito às variáveis pesquisadas.

O NAF inicial dos participantes foi documentado na pesquisa de Freiberger e Menz $z^{23}$,usando-se uma medida objetiva de caminhar rapidamente 8 metros e o número de horas auto-relatadas de caminhada por semana. A história de quedas registrada foi a dos últimos seis meses (32\% dos sujeitos relataram queda). $\mathrm{O}$ monitoramento via telefone da ocorrência de queda durante os 12 meses de pesquisa foi realizado (42\% relataram queda e destes, 21\% múltiplas quedas). Neste estudo, enquanto as mulheres caíram dentro de casa, à tarde ou à noite, os homens caíram fora de casa, de manhã ou ao meio dia, nas horas de lazer. Conquanto tenham sido propostos, no início do estudo, três diferentes tipos de intervenção e uma situação controle, em nenhum momento foram apresentadas no artigo comparações entre os grupos, nem conclusões a respeito. Os resultados se baseiam em dados gerais com ênfase em pareamento de gêneros. Os autores concluíram que os idosos pesquisados eram fisicamente ativos, viviam independentemente, experimentaram altos índices de queda fora de casa enquanto desempenhavam suas atividades da vida diária, o que raramente ocasionou ferimentos ou internação.

No estudo de Hauer et al. ${ }^{24}$, o NAF foi avaliado pelo QBMI, o mesmo utilizado por Christofoletti et al. ${ }^{26}$. Como descrito anteriormente, as intervenções Treinamento de Resistência Progressiva (TRP) e Atividade Placebo (AP) foram comparadas. As avaliações das variáveis estudadas foram feitas três a quatro semanas depois da admissão em um hospital de reabilitação (T1), no final do período de treinamento (T2), após três meses (T3) e dois anos de seguimento (T4). Nas medidas iniciais, todos os participantes tiveram história de algum tipo de ferimento relacionado à queda, nos últimos três meses, a maioria por fraturas em membros inferiores ou quadril e por outras razões como dores ou prejuízos motores. No período de intervenção, um significativo incremento do desempenho funcional no TRP foi paralelo ao aumento da atividade física, mas ambos os grupos apresentaram redução no desempenho funcional, o que foi associado à diminuição do nível de atividade no decorrer do período de seguimento. Constatou-se que sem um suporte externo, os idosos do grupo TRP retornaram ao seu estilo de vida sedentário habitual.

De maneira geral, conforme os estudos encontrados, houve uma associação entre nível de atividade física e fatores associados à ocorrência de quedas. Dentre esses fatores estão a melhora da capacidade funcional, da qualidade de vida, independência nas atividades da vida diária e redução do medo de cair. Ficou evidente em algumas pesquisas que o evento da queda ou o seu medo levam à restrição de atividades físicas, principalmente em idosos mais velhos.

\section{CONSIDERAÇÕES FINAIS}

Devido às limitações de alguns dos estudos, quanto à não representatividade das amostras, a ausência de grupo controle, heterogeneidade dos instrumentos de aferição do NAF e - no caso de estudos experimentais - acompanhamento dos participantes do estudo após o término da intervenção por um determinado período, não foi possível identificar se maiores níveis de atividade física são capazes de reduzir a incidência de quedas. Apenas um estudo selecionado fez este acompanhamento, todavia, seus resultados somente poderiam ser generalizados para idosos com saúde muito fragilizada, pelas características da população analisada, e não para idosos da população em geral que também experimentam quedas acidentais.

Como o objetivo de uma revisão sistemática é ter evidências suficientes sobre um tema específico para tomadas de decisões acertadas no campo profissional ou para o desenvolvimento do conhecimento do referido assunto, sugerimos novos estudos que utilizem métodos adequados que possam reduzir as limitações supracitadas.

\section{REFERÊNCIAS BIBLIOGRÁFICAS}

1. Masud T, Morris RO. Epidemiology of falls. Age and ageing 2001;30(4):3-7.

2. Perracini MR, Ramos LR. Fatores associados à queda em uma coorte de idosos residentes na comunidade. Rev Saúde Pública 2002;36(6):709-716.

3. Suzuki, M, Ohyama N, Yamada K, Kanamori M. The relationship between fear of falling, activities of daily living and quality of life among elderly individuals. Nurs Health Sci 2002;4(4):155-161.

4. Laessoe U, Hoeck HC, Simonsen O, Sinkjaer T, Voigt $\mathrm{M}$. Fall risk in an elderly population - can it be assessed? J Negat Results Biomed 2007;6(2):1-11. 
5. Pate RR, Pratt M, Blair SN, Haskell WL, Macera CA, Bouchard C et al. Physical activity and public health: a recommendation from the centers for disease control and prevention and the American College of Sports Medicine; JAMA 1995;273(5):402-407.

6. Gillespie LD, Gillespie WJ, Robertson MC, Lamb SE, Cumming RG, Rowe BH. Intervenciones para la prevención de caídas en las personas ancianas (cochrane review). In: la biblioteca cochrane plus. 2007; issue 3.

7. Northridge ME, Nevitt MC, Kelsey JL, Link B. Home hazards and falls in the elderly: the role of health and functional status. Am J Public Health 1995;85(4):509-515.

8. Li W, Keegan, THM, Sternfeld B, Sidney S, Quesenberry Jr CP, Kelsey,JL. Outdoor falls among middle-aged and older adults: a neglected public health problem. Am J Public Health 2006;96(7):1192-11200.

9. Jorstad-Stein EC, Hauer K, Becker C, Bonnefoy M, Nakash RA, Skelton DA, et al. Suitability of physical activity questionnaires for older adults in fall-prevention trials: a systematic review. J Aging Phys Act 2005;13(4):461-481.

10. Brasil. Ministério da saúde. Estatuto do idoso. 2 ed. Brasília (Brazil): Editora do ministério da saúde; 2006. (Série e Legislação de Saúde).

11. Freitas EV. Atividade física e o envelhecimento saudável. In: Pacheco JL, editor. Tempo: rio que arrebata. Holambra; 2005. p.107-120.

12. Farias GM. Deficiências, incapacidades e desvantagens decorrentes de causas externas: análise em pacientes internados no IOT-HCFMUSP. [Dissertation]. Säo Paulo (Brazil): Universidade de Säo Paulo. Escola de Enfermagem; 1991

13. Matsudo SM, Matsudo VKR. Prescriçäo e benefícios da atividade física na terceira idade. Rev Bras Ciênc Mov 1992;6(4):19-30.

14. Buchner DM. Preserving mobility in older adults. West J Med 1997;167(4):258-264.

15. Takekuma, K, Ishikawa H, Hayase S, Kuno K, Tsushita $\mathrm{K}$, Tominaga S. Utility of cycle ergometer stress testing as a measure to assess health status and physical functioning for elderly people. Nippon koshu Eisei Zasshi 2005;52(6):468-476.

16. Becker C. Exercise promotion in the elderly: mobility is not a goal by itself (bewegungsförderung bei alten menschen: mobilität ist kein selbstzweck). Pflege Z 2004;57(1):774-775.

17. Blain H, Vuillemin A, Blain A, Jeandel C. The preventive effects of physical activity in the elderly (les effets préventifs de l'activité physique chez les personnes agées). Presse Méd 2000;29(22):1240-1248.

18. Vance DE, Ball KK, Roenker DL, Wadley VG, Edwards JD, Gissell GM. Predictors of falling in older maryland drivers: a structural-equation model. J Aging Physical Act 2006;14(3):254-269.

19. Cipher DJ, Clifford PA, Roper KD. Behavioral manifestations of pain in the demented elderly. J Am Med Dir Assoc 2006;7(6):355-365.

20. Bergland A, Pettersen AM, Laake K. Functional status among elderly norwegian fallers living at home. Physioth Res Int 2000;5(1):33-45.
21. Bryant E, Trew M, Bruce A. Case report: activity after retirement. Physiother Res Int 2006;11(1):51-55.

22. Lin MR, Wolf SL, Hwang HF, Gong SY, Chen CY. A randomized, controlled trial of fall prevention programs and quality of life in older fallers. J. Am Geriatr Soc 2007;55(4):499-506.

23. Freiberger E, Menz HB. Characteristics of falls in physically active community-dwelling older people. Z Gerontol Geriatr 2006;39(4):261-267.

24. Hauer K, Pfisterer M, Schuler M, Bartsch P, Oster P. Two years later: a prospective long-term follow-up of a training intervention in geriatric patients with a history of severe falls. Arch Phys Med Rehabil 2003;84(10):1426-1432.

25. Sampaio RF, Mancini MC. Estudos de revisão sistemática: um guia para síntese criteriosa da evidência científica. Rev Bras fisioterap 2007;11(1):83-89.

26. Christofoletti G, Oliani MM, Gobbi LTB, Gobbi S, Stella F. Risco de quedas em idosos com doença de parkinson e demência de alzheimer: um estudo transversal. Rev Bras fisioter 2006;10(4):429-433.

27. Fone S, Lundgren-Lindquist B. Health status and functional capacity in a group of successfully ageing 65-85-year-olds. Disabil Rehabil 2003;25(18):10441051.

28. Morris M, Osborne D, Hill K, Kendig H, LundgrenLindquist B, Browning $\mathrm{C}$, et al. Predisposing factors for occasional and multiple falls in older australians who live at home. Aust J Physiother 2004;50(3):153-159.

29. Spirduso WW. Dimensões físicas do envelhecimento. Barueri: Manole; 2005.

30. Lamonte MJ, Ainsworth BE. Quantifying energy expenditure and physical activity in the context of dose response. Med Sci Sports Exerc 2001;33(6):370-378.

31. Chou KI, Yeung FK, Wong EC. Fear of falling and depressive symptoms in chinese elderly living in nursing homes: fall efficacy and activity level as mediator or moderator? Aging Ment Health 2005;9(3):255-261.

32. Ruthig JC, Chipperfield JG, Newall NE, Perry RP, Hall NC. Detrimental effects of falling on health and well-being in later life: the mediating roles of perceived control and optimism. J. Health psychol 2007;12(2):231-248.

33. Fabrício SCC, Rodrigues, RAP, Costa Junior ML. Causas e conseqüências de quedas de idosos atendidos em hospital público. Rev Saúde Pública 2004;38(1):93-99.

\footnotetext{
Endereço para correspondência

Priscila Carneiro Valim-Rogatto.

Caixa postal 192

CEP: 37200-970. Lavras, MG. Brasil.

E-mail: lappex@yahoo.com.br
} 